\title{
Challenges for the Future of Complementary and Integrative Care
}

\section{Chenchen Wang*}

Associate Professor of Medicine, Director of the Center for Complementary and Integrative Medicine, Tufts Medical Center/ Tufts University School of Medicine,Boston, MA, USA

Over the past decade, a cascade of projects and initiatives on complementary and integrative medicine has erupted globally, culminating in thousands of new scientific publications and a flood of discoveries in healthcare and health sciences.

Indeed, patients suffering from chronic conditions seek other effective treatments like complementary and alternative medicines in addition to conventional medications, for symptom management, including mind-body practice, herbal remedies, acupuncture, and various supplements.

It is estimated that over $40 \%$ of American people integrate one or more of these unconventional health practices, while many individuals worldwide also explore multidimensional approaches as treatment for chronic conditions beyond pharmacological therapies. For example, chronic conditions such as arthritis are among the top 6 conditions for complementary and alternative medicine use [1]. In fact, overwhelming evidence suggests that specific elements of complementary, alternative and integrative medicine, including mind-body approaches and improved lifestyle-modification strategies, may prevent disease, relieve pain, and enhance both physical and psychological health [28]. Individualized treatment that embraces an integrative approach may positively affect the progression of disease while simultaneously decreasing morbidity.

Although recent advances in complementary, alternative and integrative medicine have deepened our knowledge and focused our perception of medical treatment, the impacted healthcare practices which remain, continue to face formidable challenges. These challenges include: First- the complex and confounding, multifaceted and multivariable factors of complementary, alternative and integrative medicine systems require constant innovation to achieve rigorous, welldesigned studies; the current models for understanding biomedicine limit complementary, alternative and integrative medicine research and need to be expanded and extended. Second, the exploration and eventual discovery of plausible scientific mechanisms, theoretical and historical investigations are essential in order to further and fully understand the holistic role and claims of complementary, alternative and integrative medicine within the context of western medicine. Third, we are compelled to address the critical unmet needs of novel effective CAM treatments for patients who struggle with toxicity and increasing costs of their medications. Fourth, generating critical insights into comparative clinical effectiveness research to provide optimal treatments for patients is crucial, especially for long term outcomes. Finally, high quality public education which will define the use of complementary and alternative research expertise and promote effective leadership needs to be strengthened. So it is, that after a brief two decades of forceful efforts from National Center for Complementary and Alternative Medicine at National Institute of Health on behalf of complementary and alternative research, there remain insuperable tasks for scientists in evaluating thousands of years' worth of clinical research questions to prove the efficacy of complementary and alternative medicine and to better provide evidence-based, patient-centered care.

Despite these challenges, we are poised presently at the start of a major paradigm shift in health care, a change in strategic thinking which will inevitably tilt quickly towards personalized medicine to optimize health and develop individualized therapy and selfmanagement to combat disease. To accelerate this process, active collaboration with multi-disciplinary teams who can lead high-quality, original research is essential to ultimately inform and improve better clinical decision-making. In addition, targeted or individualized treatment that welcomes and embraces an integrative approach offers the undeniable potential to positively affect the progression of disease while simultaneously diminishing pain and morbidity. The demand for effective complementary and integrative treatment options will continue to grow beyond the already important demands of today.

The $21^{\text {st }}$ century promises to be the most exciting era in medicine. Modern science abetted by modern technology provides us with an unprecedented opportunity to explore in depth the mysteries, complexities, and perplexities of the human body and mind. As we broaden our understanding of health, the concepts of disease and wellbeing will also widen and deepen, and we will be able, with experience and learning, to investigate and explore all the options for integrative western and eastern medicines for symptom management while improving physical and psychological health and wellbeing.

By empowering the brightest available scientific minds and renewable resources, we can improve the prospects of the evolving treatment paradigm embedded in current healthcare and transform medicine, thereby paving the way for cost effective cures and improving quality of life in ways that are affordable, sustainable, and equitable.

\section{References}

1. Barnes P, Powell-Griner E, McFann K, Nahin R (2004) Advance data from Vital health statistics of the National Center for Health Statistics, Hyattsville.

2. Briggs JP, Killen J (2013) Perspectives on Complementary and Research JAMA 310: 691-692.

3. Wang C, Collet J, Lau J (2004)The Effect of Tai Chi on Health Outcomes in Patients with Chronic Conditions: A Systematic Review. Archive Intern Med 164: 493-501.

4. Wang C, Schmid C, Kalish R, Yinh J, Rones R, et al. (2010) Tai Chi is effective in treating fibromyalgia: a randomized controlled trial. N Engl J Med 363: 743754

*Corresponding author: Chenchen Wang, Division of Rheumatology,Tufts Medical Center, Box 406, Tufts University School of Medicine, Boston, MA 02111, USA, Tel: 617-636-3251; Fax: 617-636-1542; E-mail: cwang2@tuftsmedicalcenter.org

Received February 5, 2014; Accepted February 4, 2014; Published February 9, 2014

Citation: Wang C (2014) Challenges for the Future of Complementary and Integrative Care. Health Care Current Reviews 2: e102. doi: 10.4172/2375-4275.1000e102

Copyright: (C) 2014 Wang C. This is an open-access article distributed under the terms of the Creative Commons Attribution License, which permits unrestricted use, distribution, and reproduction in any medium, provided the original author and source are credited. 
5. Wang C, Bannuru R, Ramel J, Kupelnick B, Scott T, et al. (2010) Tai Chi on psychological well-being: systematic review and meta-analysis.BMC Complement Altern Med 23: 1186-1472.

6. Wang C (2012) Role of Tai Chi in the Treatment of Rheumatologic Diseases. Curr Rheumatol Rep 14: 598-603.
7. Wang C (2013) Complementary and Alternative Medicine and Osteoarthritis. Int Integrative Med13:1-5.

8. Wang C (2012) Complementary and Alternative Medicine in the Treatment of Rheumatoid Arthritis. Rheumatoid Arthritis - Treatment. 